\title{
Effect of pressure on the valency of cerium in cerium monochalcogenides and cerium monopnictides
}

\author{
Md. Tarek Hossain ${ }^{1}$, Abdul Hannan ${ }^{1 *}$ and Sultana Rokeya Parvin ${ }^{2}$ \\ ${ }^{1}$ Department of Physics, School of Physical Sciences, Shahjalal University of Science and Technology, Sylhet-3114, Bangladesh. \\ ${ }^{2}$ Govt. Agragami Girls' High School and College, Sylhet, Bangladesh.
}

\begin{abstract}
Application of pressure on a material changes its properties due to the modification of the electronic states of the constituent atoms. Various effects of pressure on the properties of solid material have been identified, and valency change of an element in a compound is one of the effects of pressure. This study was aimed at finding the valency change as a function of pressure of some compounds. The valency changes of cerium in cerium monochalcogenides $\mathrm{CeX}(\mathrm{X}=\mathrm{O}$ and $\mathrm{S})$ and cerium monopnictides $\mathrm{CeX}(\mathrm{X}=\mathrm{P}$ and $\mathrm{As})$ as functions of pressure have been calculated utilising the reported data. For calculating the valency change of $\mathrm{Ce}$, the reported experimental pressure-volume relationships of $\mathrm{CeO}, \mathrm{CeS}, \mathrm{CeP}$ and CeAs have been reproduced using the Birch equation of state. The corresponding pressure-volume relationships of these compounds for stable trivalent cerium have also been calculated using the same Birch equation. The change in valency is calculated by a technique, which considers the difference between the calculated and experimental lattice parameters at each pressure value of the concerned compound. Clear effect of pressure on the cerium valency has been obtained for each compound from the present calculation.
\end{abstract}

Keywords: Birch equation of state, cerium monochalcogenides, cerium monopnictides, valency change.

\section{INTRODUCTION}

The application of hydrostatic pressure on a solid material has a direct effect on the extra-nuclear electronic states of the constituent atoms in the material. Various effects of pressure such as structural phase transition (Léger, 1993), unusual crystal lattice behaviour (Iwasa et al., 1999; Hannan et al., 2000), unusual electrical resistivity
(Okayama et al., 1992) and the appearance of complicated magnetic phase (Hannan et al., 2002; Osakabe et al., 2002) have been reported.

In this study, we have focused on cerium monochalcogenides $\mathrm{CeX}(\mathrm{X}=\mathrm{O}, \mathrm{S}, \mathrm{Se}$ and $\mathrm{Te})$ and cerium monopnictides $\mathrm{CeX}(\mathrm{X}=\mathrm{P}, \mathrm{As}, \mathrm{Sb}$ and $\mathrm{Bi})$. Léger et al. (1987) have investigated the pressure-volume relationship and the crystallographic phase changes of these compounds by X-ray diffraction in diamond anvil cells at room temperature (Vedel et al., 1986; Léger et al., 1987; Léger, 1993). These compounds exhibit an unusual pressure-volume relationship in the wide pressure range from 0 to about $25 \mathrm{GPa}$. The pressure-volume relationships of $\mathrm{CeX}(\mathrm{X}=\mathrm{O}, \mathrm{S}, \mathrm{Se}$ and $\mathrm{Te})$ have been reported by Léger (1993). The pressure-volume relationships of some selected compounds from both cerium monochalcogenides and monopnictides have been investigated using the Birch equation of state (Birch, 1947; Mito et al., 2007) to calculate the valency change as a function of pressure (details are presented later in the text). In cerium monochalcogenides $\mathrm{CeX}$ $(\mathrm{X}=\mathrm{O}, \mathrm{S}, \mathrm{Se}$ and $\mathrm{Te}$ ), the volume of $\mathrm{CeO}$ decreases very rapidly with pressure up to $3 \mathrm{GPa}$, above which the rate of change exhibits a normal trend (Léger, 1993). The crystal structure of this compound up to $25 \mathrm{GPa}$ remains the same (NaCl-type). The volume of $\mathrm{CeS}$ decreases smoothly up to a pressure of $27 \mathrm{GPa}$ exhibiting the $\mathrm{NaCl}$-type structure. CeSe also shows the $\mathrm{NaCl}$-type phase up to $25 \mathrm{GPa}$ but above $20 \mathrm{GPa}$ a phase of CsCl-type structure appears with a volume change of $9 \%$. At $8 \mathrm{GPa}$, CeTe changes the structural phase from NaCl-type to CsCl-type (Léger, 1993). 
There are also reported works on the relative volume of cerium monopnictides $\mathrm{CeX}(\mathrm{X}=\mathrm{P}, \mathrm{As}, \mathrm{Sb}$ and $\mathrm{Bi})$ as a function of pressure (Léger, 1993). CeP shows isostructural discontinuous transition at around $10 \mathrm{GPa}$, and above $20 \mathrm{GPa}$ it exhibits a structural transition from $\mathrm{NaCl}$-type to a CsCl-type structure (Vedel et al., 1987; Léger, 1993). CeAs shows a sharp structural transition from $\mathrm{NaCl}$-type to CsCl-type at about $18 \mathrm{GPa}$. CeSb exhibits a sharp structural transition from NaCl-type to body central tetragonal structure at $11 \mathrm{GPa}$. At $13 \mathrm{GPa}$, $\mathrm{CeBi}$ exhibits structural transition from $\mathrm{NaCl}$-type to CsCl-type structure.

Crystal-lattice behaviour of $\mathrm{CeSb}$ has been extensively studied earlier (Hannan et al., 2000) under various high pressures using a diamond-anvil cell (DAC). They observed an unusual shrinking of the crystal lattice below a certain critical temperature $T_{\mathrm{L}}$ in the paramagnetic region, which nearly corresponds to the resistivity anomaly temperature $T_{\mathrm{R}}$ as reported earlier (Okayama et al., 1992).

Iwasa et al. (1999) measured the temperature dependence of the lattice constants of $\mathrm{CeP}$ and CeAs by $\mathrm{X}$-ray diffraction. The lattice constants of $\mathrm{CeP}$ and $\mathrm{CeAs}$ show clear minima at $120 \mathrm{~K}$ and $90 \mathrm{~K}$, respectively. These temperatures are close to the crystal field splitting energies of $160 \mathrm{~K}$ and $150 \mathrm{~K}$ of $\mathrm{CeP}$ and $\mathrm{CeAs}$, respectively. Unusual temperature dependencies of the lattice constants in $\mathrm{CeP}$ and $\mathrm{CeAs}$ have been explained, assuming that the interatomic bond length between cerium ions of $\Gamma_{8}$ state and pnictogens is shorter than that of $\Gamma_{7}$ cerium ions (Iwasa et al., 1999).

$\mathrm{CeSb}$ shows a complicated magnetic pressuretemperature phase diagram (Chattopadhyay et al., 1994; Osakabe et al., 2002). The magnetic pressure-temperature phase diagram of $\mathrm{CeP}$ is more complicated than that of $\mathrm{CeSb}$ (Hannan et al., 2002). The resistivity of CeP exhibits a surprisingly pressure sensitive behaviour (Okayama et al., 1992). At ambient pressure, the sharp pointed peak at about $10 \mathrm{~K}$ is due to the antiferromagnetic phase transition. The resistivity passes through a minimum at about $23 \mathrm{~K}$, a broad maximum at around $80 \mathrm{~K}$ and then it decreases with increasing temperature. This is a typical magnetic dense Kondo behaviour. At ambient pressure, there is a sharp pointed peak for CeAs corresponding to the Néel temperature $7.7 \mathrm{~K}$ (Okayama et al., 1992). A broad maximum is also observed at around $60 \mathrm{~K}$ with a Kondo type behaviour in the higher temperature region. Enormous enhancement of the electrical resistivity of $\mathrm{CeSb}$ is seen near $30 \mathrm{~K}$ at pressures above $3 \mathrm{GPa}$. The peak value of resistivity increases with increasing pressure, and at $7 \mathrm{GPa}$ it reaches a value nearly 23 times as large as that at the ambient pressure (Okayama et al., 1992). $\mathrm{CeBi}$ exhibits a sharp pointed peak near $30 \mathrm{~K}$ associated with the magnetic phase. At pressures higher than $3 \mathrm{GPa}$, however, the sharp pointed peak changes into a hump and shows a tendency to disappear gradually with increasing pressure (Okayama et al., 1992). All of the features mentioned above have been driven by the application of various hydrostatic pressures on the compounds.

In this study the valency change of $\mathrm{Ce}$ in some compounds of cerium monochalcogenides $\mathrm{CeX}$ $(\mathrm{X}=\mathrm{O}, \mathrm{S}, \mathrm{Se}$ and $\mathrm{Te})$ and cerium monopnictides $\mathrm{CeX}(\mathrm{X}=\mathrm{P}, \mathrm{As}, \mathrm{Sb}$ and $\mathrm{Bi})$ with the change of pressure has been calculated. In doing this, the reported (Léger, 1993) experimental pressure-volume relationships of the compounds have been reproduced by using the Birch equation of state. The corresponding pressure-volume relationships of these compounds for stable trivalent cerium have also been calculated using the same Birch equation. The clear effect of pressure on the cerium valency has been realised for each compound from the present calculation.

\section{CALCULATION OF VALENCY CHANGE DUE TO PRESSURE}

\section{Reproduction of experimental relative volume}

The experimental pressure-volume relationship, as reported by Léger (1993), can be fitted using the Birch equation of state (Birch, 1947; Mito et al., 2007) as written below:

$P(y)=\frac{3}{2} B_{0}\left(\mathrm{y}^{-7 / 3}-y^{-5 / 3}\right)\left[1-\frac{3}{4}\left(B^{*}-4\right)\left(1-y^{-2 / 3}\right)\right]$,

where $P$ is the pressure, $y=V / V_{0}$ is the relative volume, $B_{0}$ is the Bulk modulus and $B^{*}$ is the pressure derivative of the Bulk modulus. The values of $B_{0}$ and $B^{*}$ come as fitting parameters. So if the fitting parameters are known, the experimental trend (data) of the relative volume as a function of pressure can be reproduced.

The reproduced experimental relative volumes for $\mathrm{CeO}$ and $\mathrm{CeS}$ are shown in Figure 1 by solid triangles and solid diamonds, respectively. The values of the parameters $B_{0}$ and $B^{*}$ used for $\mathrm{CeO}$ are $30 \mathrm{GPa}$ and 5 , and for CeS are $82 \mathrm{GPa}$ and 2.25, respectively (Léger, 1993). The calculation for the reproduction of the experimental data has been done by IGOR Pro software (Version 4.0). 


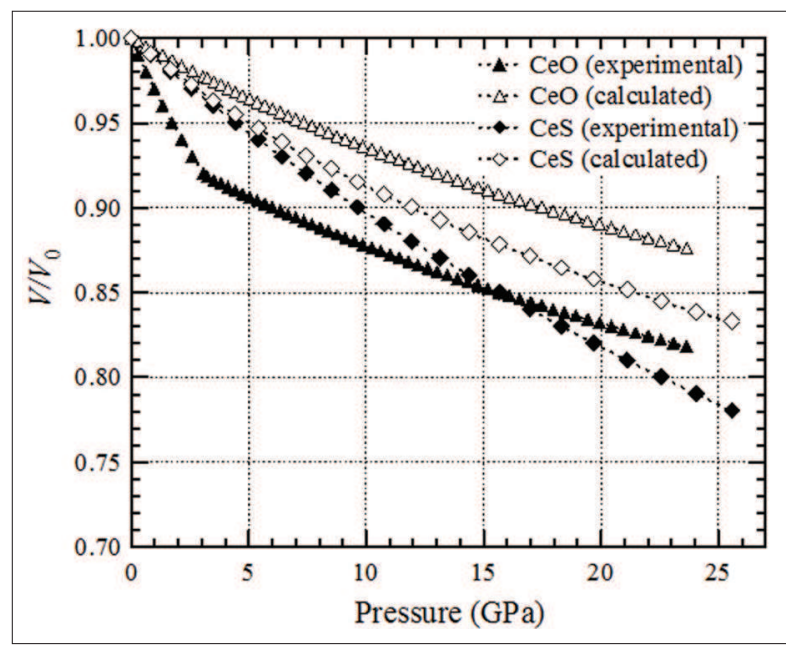

Figure 1: Reproduced experimental pressure-volume curves of $\mathrm{CeO}$ and $\mathrm{CeS}$ (solid triangles and solid diamonds) and the corresponding calculated curves (open triangles and open diamonds) for stable trivalent cerium in the compounds.

The reproduced experimental relative volumes for CeP and CeAs using equation (1) are shown in Figure 2 by solid squares and solid circles, respectively. The values of the parameters $B_{0}$ and $B^{*}$ used for $\mathrm{CeP}$ are $64 \mathrm{GPa}$ and 3, and for CeAs are $69 \mathrm{GPa}$ and 2.6, respectively (Léger, 1993).

\section{Theoretical relative volume for stable trivalent cerium}

Two compounds, $\mathrm{CeO}$ and $\mathrm{CeS}$, from the category of cerium monochalcogenides have been selected. For trivalent stable cerium in $\mathrm{CeO}$ the parameters $B_{0}$ and $B^{*}$ are $125 \mathrm{GPa}$ and 5.4, and in CeS they are $85 \mathrm{GPa}$ and 5.4, respectively (Léger, 1993). Using these parameters in equation (1), the relative volumes of $\mathrm{CeO}$ and $\mathrm{CeS}$ as a function of pressure have been calculated. The calculated relative volumes are presented in Figure 1 by open triangles and open diamonds.

For trivalent stable cerium in $\mathrm{CeP}$ the parameters $B_{0}$ and $B^{*}$ are $78 \mathrm{GPa}$ and 5.4, and in CeAs they are $72.44 \mathrm{GPa}$ and 5.4, respectively (Léger, 1993). The relative volumes of $\mathrm{CeP}$ and $\mathrm{CeAs}$ as a function of pressure have been calculated using equation (1). The calculated relative volumes are presented in Figure 2 by open squares and open circles.

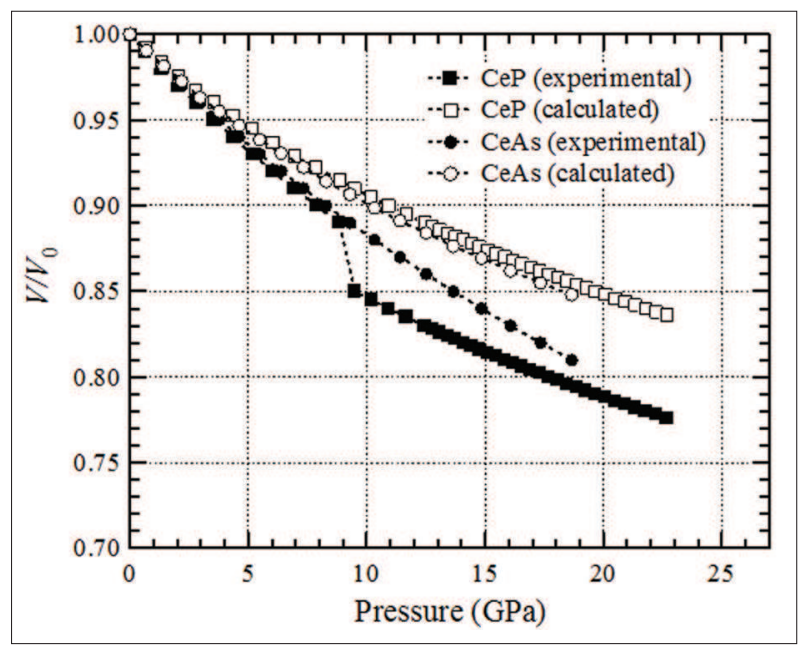

Figure 2: Reproduced experimental pressure-volume curves of $\mathrm{CeP}$ and CeAs (solid squares and solid circles) and the corresponding calculated curves (open squares and open circles) for stable trivalent cerium in the compounds.

\section{Calculation of valency change}

It is assumed that the valency of cerium in cerium monochalcogenide and cerium monopnictide compounds varies linearly with the lattice parameter, $a$ (Léger, 1993). The increase of the valency, $x$ above $3+$ can be calculated by the following relation.

$$
x=\frac{\left\{a_{\text {calc }}^{3+}(P)-a_{\text {exp }}^{3+x}(P)\right\}}{\left\{a_{\text {calc }}^{3+}\left(P_{0}\right)-a_{\text {calc }}^{4+}\left(P_{0}\right)\right\}},
$$

where $a_{\text {calc }}^{3+}(P)$ is the calculated lattice constant at pressure $P$ with cerium valency $3+, a_{\exp }^{3+x}(P)$ is the experimentally observed lattice constant at pressure $P$ with cerium valency $3+\mathrm{x}, a_{\text {calc }}^{3+}\left(P_{0}\right)$ is the calculated lattice constant at ambient pressure with cerium valency $3+$ and $a_{\text {calc }}^{4+}\left(P_{0}\right)$ is the calculated lattice constant at ambient pressure with cerium valency $4+$.

In the calculation, the cerium coordination must be taken into account and the following cerium radii with a coordination of six, for $f c c$ structure, are used: $r_{\mathrm{Ce}}^{3+}\left(P_{0}\right)=1.034 \AA, r_{\mathrm{Ce}}^{4+}\left(P_{0}\right)=0.85 \AA$ (Léger, 1993). 
So, for $\mathrm{NaCl}$-type cubic lattice we can write

$\underset{\text { calc }}{a^{3+}}\left(P_{0}\right)-a_{\text {calc }}^{4+}\left(P_{0}\right)=2\left\{r_{\mathrm{Ce}}^{3+}\left(P_{0}\right)-r_{\mathrm{Ce}}^{4+}\left(P_{0}\right)\right\}$

$=2(1.034-0.85) \AA \dot{=}=0.368 \AA$

So, equation (2) becomes

$x=\frac{\left\{a_{\text {calc }}^{3+}(P)-a_{\text {exp }}^{3+x}(P)\right\}}{0.368}$

or $\quad x=\frac{a_{\text {calc }}^{3+}\left(P_{0}\right)\left\{\frac{a_{\text {calc }}^{3+}(P)}{a_{\text {calc }}^{3+}\left(P_{0}\right)}-\frac{a_{\text {exp }}^{3+x}(P)}{a_{\text {calc }}^{3+}\left(P_{0}\right)}\right\}}{0.368}$

where $a_{\text {calc }}^{3+}\left(P_{0}\right)$ is the lattice parameter of the concerned $f c c$ lattice at ambient pressure.

or $x=\frac{a_{\text {calc }}^{3+}\left(P_{0}\right)\left[\left\{\frac{V_{\text {calc }}(P)}{V\left(P_{0}\right)}\right\}^{1 / 3}-\left\{\frac{V_{\text {exp }}(P)}{V\left(P_{0}\right)}\right\}^{1 / 3}\right]}{0.368}$,

where $V_{\text {calc }}(P)=\left\{a_{\text {calc }}^{3^{+}}(P)\right\}^{3}$ is the calculated volume of the unit cell at pressure $P$ with cerium valency $3+$, $V_{\text {exp }}(P)=\left\{a_{\exp }^{3+x}(P)\right\}^{3}$ is the experimentally observed volume of the unit cell at pressure $P$ with cerium valency $3+x$ and $V\left(P_{0}\right)=\left\{\underset{\text { calc }}{a^{3+}}\left(P_{0}\right)\right\}^{3}$ is the calculated volume of the unit cell at ambient pressure with cerium valance $3+$.

The valency of Ce at pressure $P$ is calculated from the following relation:

Valency $=3+\frac{a_{\text {calc }}^{3+}\left(P_{0}\right)\left[\left\{\frac{V_{\text {calc }}(P)}{V\left(P_{0}\right)}\right\}^{1 / 3}-\left\{\frac{V_{\text {exp }}(P)}{V\left(P_{0}\right)}\right\}^{1 / 3}\right]}{0.368}$

\section{RESULTS AND DISCUSSION}

The effect of pressure on the valency change of some compounds is presented in this article. In Figures 1 and 2 , there is a big difference between the experimental and calculated pressure-volume curves. This difference is due

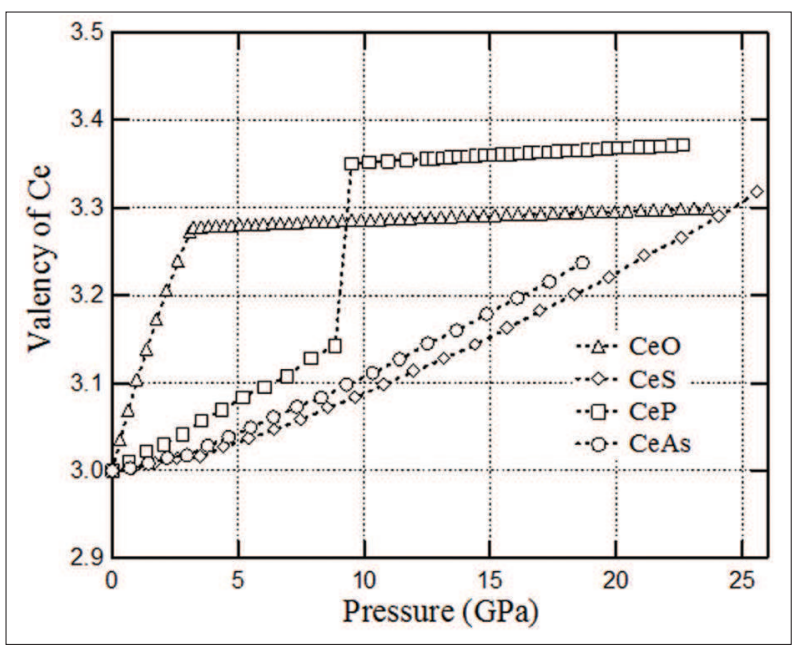

Figure 3: Valency of $\mathrm{Ce}$ in $\mathrm{CeO}, \mathrm{CeS}, \mathrm{CeP}$ and $\mathrm{CeAs}$.

to the enhancement of valency above $3+$ with pressure, as can be seen from equation (6). Figure 3 shows the valency of $\mathrm{Ce}$ calculated using equation (6), in $\mathrm{CeO}, \mathrm{CeS}, \mathrm{CeP}$ and $\mathrm{CeAs}$ as a function of pressure. For $\mathrm{CeO}$, the valency of Ce rapidly increases with the pressure, and at about $3 \mathrm{GPa}$ the valency is $3.27+$. Above $3 \mathrm{GPa}$ the valency is almost pressure independent up to $23.5 \mathrm{GPa}$. The valency of $\mathrm{Ce}$ in $\mathrm{CeS}$ increases non-linearly with the pressure, and at about $25.5 \mathrm{GPa}$ the valency is $3.31+$. For $\mathrm{CeP}$, the valency of $\mathrm{Ce}$ increases almost linearly with the increase of pressure, and at $9 \mathrm{GPa}$ the valency becomes $3.14+$. Above this pressure, the valency suddenly increases to $3.35+$ at about $9.5 \mathrm{GPa}$ due to isostructural valence-phase transition, and then the valency is almost pressure independent up to $22.5 \mathrm{GPa}$. In the case of CeAs, a continuous non-linear increase of valency with the increase of pressure is found, and at $18.5 \mathrm{GPa}$ the $\mathrm{Ce}$ valency is $3.23+$. It is seen that in any group of compounds, the valency change does not depend on the group, but it is dependent on the particular sample of the group.

\section{CONCLUSION}

In this study a method adopting the Birch equation of state has been introduced for the calculation of valency change of cerium in $\mathrm{CeO}, \mathrm{CeS}, \mathrm{CeP}$ and CeAs. Our calculation shows a clear effect of pressure on the valency change, and the maximum valency change of $0.37+$ is found for the change of pressure of 22.6 GPa for CeP. The valency change of cerium with pressure in any compound under a certain group is found to be independent of the group. That means, the environment around the $\mathrm{Ce}$ in a compound is solely responsible for the change of the valency of $\mathrm{Ce}$ in the compound. 


\section{REFERENCES}

1. Birch F. (1947). Finite elastic strain of cubic crystals. Physical Review 71: $809-824$.

DOI: http://dx.doi.org/10.1103/PhysRev.71.809

2. Chattopadhyay T., Burlet P., Rossat-Mignod J., Bartholin H., Vettier C. \& Vogt O. (1994). High-pressure neutron and magnetization investigations of the magnetic ordering in CeSb. Physical Review B 49(21): 15096 - 15104. DOI: http://dx.doi.org/10.1103/PhysRevB.49.15096

3. Hannan A., Iwasa K., Kohgi M. \& Suzuki T. (2000). Crystal-lattice anomaly of $\mathrm{CeSb}$ under high pressure induced by magnetic polaron formation. Journal of the Physical Society of Japan 69(7): 2358 - 2359.

DOI: http://dx.doi.org/10.1143/JPSJ.69.2358

4. Hannan A., Osakabe T., Kohgi M. \& Iwasa K. (2002). High-pressure magnetic phase diagram of CeP studied by neutron diffraction. Applied Physics A 74: [Suppl.] S565 - S567.

DOI: http://dx.doi.org/10.1007/s003390201608

5. IGOR Pro Version 4.0, WaveMetrics Inc., PO Box 2088, Lake Oswego, OR 97035, USA.

6. Iwasa K., Kohgi M., Ohsumi H., Tajama K., Takeuchi T., Haga Y., Uesawa A. \& Suzuki T. (1999). X-Ray diffraction study on thermal properties of crystal lattices in $\mathrm{CeP}$ and CeAs. Journal of the Physical Society of Japan 68: $881-886$.

DOI: http://dx.doi.org/10.1143/JPSJ.68.881
7. Léger J.M., Vedel I., RedonA-M. \& Rossat-Mignod J. (1987). Pressure-volume relationship of cerium monocalcogenides and monopnictides. Journal of Magnetism and Magnetic Materials 63 \& 64: 49 - 51.

8. Léger J.M. (1993). Chalcogenides and pnictides of cerium and uranium under high pressure. Physica B 190: $84-91$.

9. Mito T., Nakamura M., Otani M., Koyama T., Wada S., Ishizuka M., Forthaus M.K., Lengsdorf R., Abd-Elmeguid M.M. \& Sarrao J.L. (2007). Magnetic properties of the pressure-induced ordering state in $\mathrm{YbInCu}_{4}$ investigated with NMR, magnetization, and x-ray diffraction measurements. Physical Review B 75: 134401(1 - 6).

10. Okayama Y., Takahashi H., Môri N., Kwon Y.S., Haga Y. \& Suzuki T. (1992). Pressure induced electrical and magnetic properties in Ce-monopnictides, $\mathrm{CeX}(\mathrm{X}=\mathrm{P}, \mathrm{As}, \mathrm{Sb}$ and Bi). Journal of Magnetism and Magnetic Materials 108: 113 - 114 .

11. Osakabe T., Hannan A., Tachi N., Kohgi M. \& Kitazawa H. (2002). Magnetic $P-T$ phase diagram and magnetic structures of CeSb. Applied Physics A 74: [Suppl.] S799 - S801. DOI: http://dx.doi.org/10.1007/s003390201586

12. Vedel I., Oki K., Redon A-M. \& Léger J.M. (1986). Electronic transitions induced by pressure in cerium monochalcogenides and monopnictides. Physica B 139 \& 140: $361-363$.

13. Vedel I., Redon A.M., Rossat-Mignod J., Vogt O. \& Léger J.M. (1987). Electronic and crystallographic transitions induced by pressure in CeP. Journal of Physics C 20: 3439. DOI: http://dx.doi.org/10.1088/0022-3719/20/23/013 